\title{
Distinct progression pathways involving the dysfunction of DUSP6/MKP-3 in pancreatic intraepithelial neoplasia and intraductal papillary-mucinous neoplasms of the pancreas
}

Toru Furukawa ${ }^{1}$, Rumi Fujisaki ${ }^{1}$, Yoshitaro Yoshida ${ }^{1}$, Naomi Kanai ${ }^{1}$, Makoto Sunamura ${ }^{2}$, Tadayoshi Abe ${ }^{1,2}$, Kazunori Takeda ${ }^{2}$, Seiki Matsuno ${ }^{2}$ and Akira Horii ${ }^{1}$

${ }^{1}$ Department of Molecular Pathology, Tohoku University School of Medicine, Sendai, Japan and

${ }^{2}$ Department of Gastroenterological Surgery, Tohoku University School of Medicine, Sendai, Japan

\begin{abstract}
DUSP6/MKP-3 is identified as a candidate tumor suppressor gene for pancreatic cancer. The aim of this study was to elucidate the roles of DUSP6 in the pancreatic carcinogenesis through the pancreatic intraepithelial neoplasia and/or intraductal papillary-mucinous neoplasms, both of which are considered to be precursor lesions of invasive carcinoma of the pancreas, by comparing with involvements of other major tumor suppressive pathways. Expressions of DUSP6, CDKN2A, TP53, and SMAD4 were investigated by immunohistochemistry in a total of 206 lesions of dysplastic ductal precursors and carcinomas retrieved from 52 pancreata with invasive ductal carcinomas and $\mathbf{5 1}$ of those with intraductal papillary-mucinous neoplasms. The intensity of staining was evaluated in lesions at different atypical grades and statistically compared among them. Mutations of KRAS2 were analyzed by methods of the allele-specific oligonucleotide hybridization and nucleotide sequencing. In pancreata with invasive ductal carcinomas, expressions of DUSP6 were abrogated exclusively in the invasive carcinoma cells in contrast to its fairly preserved expressions in pancreatic intraepithelial neoplasia. In pancreata with intraductal papillary-mucinous neoplasms, abrogated expressions of DUSP6 were observed in a relatively small fraction of intraductal adenoma/borderlines and intraductal carcinomas. Most of the intraductal adenoma/borderline lesions with abrogation of DUSP6 harbored mutations of KRAS2. None of the molecules was associated with each other in any grade of lesions. Morphological variations of papillae of the intraductal papillary-mucinous neoplasms were evaluated and analyzed for their associations with abrogations of the molecules, which resulted in finding of no significant associations. Our results suggest that the abrogation of DUSP6 is associated exclusively with progression from pancreatic intraepithelial neoplasia to the invasive ductal carcinoma while it is potentially associated with initiation of intraductal papillary-mucinous neoplasms with mutated KRAS2, which is independent of other major tumor suppressive pathways in both types of neoplasms.
\end{abstract}

Modern Pathology (2005) 18, 1034-1042. doi:10.1038/modpathol.3800383; published online 8 April 2005

Keywords: intraductal papillary-mucinous neoplasm; pancreatic intraepithelial neoplasia; DUSP6; p16; p53; SMAD4

Genomic analysis of invasive ductal carcinoma of the pancreas has revealed frequent loss of heterozygosities in several specific chromosomal regions, including 1p36, 6q21-q24, 9p21, 12q21-q23.1,

Correspondence: $\mathrm{Dr} \mathrm{T}$ Furukawa, MD, PhD, Department of Molecular Pathology, Tohoku University School of Medicine, 2-1 Seiryo-machi, Aoba-ku, Sendai, Miyagi 980-8575, Japan.

E-mail: furukawa@mail.tains.tohoku.ac.jp

Received 5 November 2004; revised and accepted 28 December 2004; published online 8 April 2005 17p13, and $18 \mathrm{q} 21 .{ }^{1-3}$ These regions potentially harbor tumor suppressor genes involved in development and progression of the pancreatic cancer. To date, several candidate tumor suppressor genes are identified in these regions, and frequent inactivating mutations have been reported in the pancreatic cancer; CDKN2A/INK4/p16 at 9p21, TP53/p53 at $17 \mathrm{p} 13$, and $S M A D 4 / M A D H 4 / D P C 4$ at $18 \mathrm{q} 21 .^{4-6}$ Recently, we identified DUSP6/MKP-3 at 12q21q22 as a candidate tumor suppressor gene. ${ }^{7,8}$ DUSP6 is a dual specificity phosphatase that intrinsically 
binds and inactivates MAPK1/ERK2 in a feedback loop manner ${ }^{9}$ and plays an important role in physiological regulation of the signaling pathway of RAS and mitogen-activated protein kinases (MAPK). ${ }^{10-13}$ In a previous report, we demonstrated that expressions of DUSP6 were reduced or abolished in cells of invasive ductal carcinoma of the pancreas in contrast to its overexpressions in dysplastic cells of pancreatic ducts. ${ }^{8}$ Cultured human pancreatic cancer cells lacking an expression of DUSP6 tended to show constitutively active MAPK1, and an adenovirus-mediated introduction of DUSP6 into such DUSP6-inactivated cells induced inactivation of MAPK1, resulting in a marked growth suppression and eventual apoptosis of the cells. $^{8}$ Since a vast majority of pancreatic cancer cells harbors the gain-of-function-mutation of KRAS2, which constitutively activates several downstream signal cascades including RAF1MAP2K1-MAPK1, these results indicated that the abrogation of DUSP6 synergistically contributes to hyperactivation of MAPK1, which may eventually result in the development and progression of invasive ductal carcinoma of the pancreas. ${ }^{9}$

Dysplastic cells in pancreatic ducts are thought to be precursor lesions of the invasive ductal carcinoma, not only because of the classic findings of their frequent distributions in pancreata involved with invasive ductal carcinomas but also because of the similarities in their molecular backgrounds that have been discovered by recent molecular studies. ${ }^{14-19}$ The recently proposed progression model based on the definition of the pancreatic intraepithelial neoplasia facilitates recognition of these dysplastic ductal regions as major precursor lesions and investigation of their molecular phenotypes. ${ }^{20,21}$ Intraductal papillary-mucinous neoplasm was first reported in 1982 as a special type of pancreatic neoplasm exhibiting a mucinous ectatic duct with dysplastic papillary epithelia. ${ }^{22}$ Intraductal papillary-mucinous neoplasms are considered to be one of the precursor lesions of the pancreatic cancer, because some patients with intraductal papillary-mucinous neoplasms eventually develop an invasive papillary-mucinous carcinoma/invasive carcinoma associated with intraductal papillarymucinous neoplasm, which is comprised of either invasive colloid mucinous carcinoma or invasive ductal carcinoma. $^{23,24}$ Although there are some morphological similarities between the pancreatic intraepithelial neoplasia and intraductal papillarymucinous neoplasms, different clinicopathological features between them suggest that the pancreatic intraepithelial neoplasia and intraductal papillary-mucinous neoplasms would develop and progress through distinct pathways involving different genetic and molecular alterations, a hypothesis which seems to be endorsed by some molecular studies. ${ }^{25-27}$

The aim of this study was to elucidate the roles of DUSP6 in the development and progression of pancreatic intraepithelial neoplasia and the intraductal papillary-mucinous neoplasm in association with other major tumor suppressive pathways. We investigated abrogations of DUSP6, CDKN2A, TP53, and SMAD4, and mutations of KRAS2 in the dysplastic ductal precursor lesions and carcinoma cells in pancreata afflicted by invasive ductal carcinomas or intraductal papillary-mucinous neoplasms.

\section{Materials and methods}

\section{Pancreatic Tissues}

Pancreatic tissues from 103 patients were analyzed in this study; 52 cases with invasive ductal carcinomas and 51 cases with intraductal papillary-mucinous neoplasms were employed in this study. Of the 52 cases with invasive ductal carcinomas, 42 were classified as tubular adenocarcinomas of the well or moderately differentiated type and 10 as adenocarcinomas of the poorly differentiated type. Of the 51 cases with intraductal papillarymucinous neoplasms, 16 tumors were intraductal papillary-mucinous adenoma/borderlines, 27 were intraductal papillary-mucinous carcinomas, five were invasive colloid mucinous carcinomas associated with intraductal papillary-mucinous neoplasms, and the remaining three were invasive ductal carcinomas associated with intraductal papillary-mucinous neoplasms. All these tissues had been surgically resected at the Department of Gastroenterological Surgery, Tohoku University Hospital. All the tissues were fixed in $10 \%$ buffered formalin and embedded in paraffin. Consecutive sections at $4 \mu \mathrm{m}$ thick were prepared; one section was stained with hematoxylin and eosin, and the others were used for immunohistochemical studies. Atypical epithelial lesions were evaluated in the sections according to the criteria published previously. ${ }^{19,21,27}$ From 52 pancreata with invasive ductal carcinoma, 38 lesions of low-grade dysplasia/pancreatic intraepithelial neoplasia-1 and -2, 24 lesions of high-grade dysplasia/pancreatic intraepithelial neoplasia-3, and 57 lesions of invasive carcinoma (47 of those of the well or moderately differentiated type and 10 of those of the poorly differentiated type) were retrieved (two or more lesions were retrieved independently from most of the patients). From 51 patients with intraductal papillary-mucinous neoplasms, 47 lesions of intraductal papillary-mucinous adenoma or borderline, 32 lesions of intraductal papillary-mucinous carcinoma, five lesions of invasive colloid mucinous carcinoma associated with intraductal papillarymucinous neoplasm, and three lesions of invasive ductal carcinoma associated with intraductal papillary-mucinous neoplasm were retrieved. Multiple kinds of lesions were retrieved independently from each case. This study was approved by the Ethical 
Committee of the Tohoku University School of Medicine.

\section{Immunohistochemistry}

Immunohistochemistry was performed by the indirect biotin-streptavidin-peroxidase method as described previously. ${ }^{8}$ The antigen retrieval method employed for staining of all molecules but DUSP6 were as described. ${ }^{28}$ The antibodies employed were a polyclonal anti-DUSP6 antibody, C-20 (Santa Cruz Biotechnology Lab Inc., Santa Cruz, CA, USA), monoclonal anti-CDKN2A antibodies, G175-405 (BD Biosciences, San Jose, CA, USA) and DCS-50 (SIGMA, St Louis, MO, USA), a monoclonal antiTP53 antibody, DO-7 (Dako Cytomation Co. Ltd, Glostrup, Denmark), a monoclonal anti-SMAD4 antibody, B8 (Santa Cruz Biotechnology Lab Inc.), a monoclonal anti-MUC1-CORE antibody, Ma552 (Novocastra Laboratories Ltd. Newcastle upon Tyne, UK), a monoclonal anti-MUC2 antibody, Ccp58 (Santa Cruz Biotechnology Lab Inc.), and a monoclonal anti-MUC5AC antibody, CLH2 (Chemicon International Inc., Temecula, CA, USA) for primary antibody reactions and biotin-conjugated anti-goat or mouse IgG $(\mathrm{H}+\mathrm{L})$ antibodies (Vector Lab. Inc., Burlingham, CA, USA) for secondary antibody reactions. The streptavidine solution (Nichirei, Tokyo, Japan) was used for a streptavidine-biotin reaction. DAB (3,3'-diaminobenzidine tetrahydrochloride) was used as a chromogen, and hematoxylin was used for counterstaining. Immunoreactivities for DUSP6, CDKN2A, TP53, and SMAD4 were graded and scored in the retrieved atypical lesions as negative $(0+)$, focally or weakly positive $(1+)$, consistently positive $(2+)$, and intensely positive $(3+)$, as described previously. ${ }^{8}$ In the evaluated expressions, $0+$ and $1+$ for DUSP6, 0 + and $1+$ for CDKN2A, $0+$ and $3+$ for TP53, and $0+$ for SMAD4 were considered as abnormal expressions, all of them suggesting abrogation of their proper functions.

\section{Analysis of KRAS2 Mutation}

Lesions were microdissected using LM100 Laser Capture Microdissection system according to the manufacturer's instructions (Arcturus, Mountain View, CA, USA). DNA was extracted by using the Pico-Pure DNA Extraction Kit according to the manufacturer's instructions (Arcturus). The allelespecific oligonucleotide hybridization method employed for analysis of mutations at cordon 12 of KRAS2 was based on the procedure described previously. ${ }^{29}$ In brief, the region harboring codon 12 of KRAS2 was amplified by PCR using specific primers reported previously, ${ }^{30}$ and the amplified product was electrophoresed in 3\% agarose gel and Southern-blotted on a nitrocellulose membrane. Oligonucleotides corresponding to all the mutations of codon 12 of KRAS2 were synthesized according to the literature, ${ }^{29}$ and $\gamma-\left[{ }^{32} \mathrm{P}\right]$ labeled probes were prepared by T4 polynucleotide kinase. Hybridization at $50^{\circ} \mathrm{C}$ in $7 \%$ PEG $10 \%$ SDS and subsequent stringent wash in $6 \times \mathrm{SSC}$ at $58-65^{\circ} \mathrm{C}$ (temperature depending on probes) was performed according to methods described previously. ${ }^{31}$ Signals were visualized by BAS-1500 according to the manufacturer's instructions (Fujifilm Co. Ltd, Minamiashigara, Japan). A control for normal KRAS2 gene (GGT/Gly at the codon 12) was prepared by PCR amplification of a normal human genomic DNA, and specific mutational patterns for the codon 12 were prepared by PCR amplification of genomic DNAs derived from cancer cell lines of PK-8 (CGT/Arg), MIA PaCa2 (TGT/Cys), SU 86.86. (GAT/Asp), PAN03JCK (GTT/Val), and A549 (AGT/Ser) based on the information reported previously. ${ }^{30,32}$ In lesions that did not show any mutation by the allele-specific oligonucleotide hybridization at codon 12 , the direct sequencing of PCR-amplified products was performed to analyze genetic alterations around codons 12,13 , and 61 of KRAS2 gene as described previously. ${ }^{30,33}$ Primers used for amplifying the genetic region around codons 12 and 13 were the same as used in the allele-specific oligonucleotide analysis. Primers used for amplifying the genetic region around codon 61 were $5^{\prime}$-CCTTCTCAG GATTCCTACAGG-3' and 5'-AAGAAAGCCCTCCC CAGTCCT-3'.

\section{Statistics}

The results of immunohistochemistry were compared statistically by the $\chi^{2}$ method. Significant difference was considered as $P<0.05$ in Fisher's exact test. Calculations were performed using Statview software (SAS Institute Inc., Cary, NC, USA).

\section{Results}

We first investigated the expression of DUSP6 along with CDKN2A, TP53, and SMAD4 by immunohistochemistry in a total of 206 lesions of dysplastic ductal precursors and carcinomas retrieved from 52 pancreata with invasive ductal carcinomas and 51 pancreata with intraductal papillary-mucinous neoplasms. In the retrieved lesions, abrogations of expressions of the molecules in pancreatic intraepithelial neoplasia and invasive ductal carcinomas are summarized in Table 1. Representative images of the immunohistochemistry are shown in Figure 1. To elucidate critical and susceptible stages for inactivations of the molecules, the frequencies of abnormal expressions were compared among the atypical grades statistically by the $\chi^{2}$ method and the difference was represented by Fisher's exact $P$-value. As indicated in Table 1 and Figure 2a, significant differences were observed in comparison 
Table 1 Abnormal expressions of DUSP6, CDKN2A, TP53, and SMAD4 in atypical ductal lesions/pancreatic intraepithelial neoplasia and invasive ductal carcinoma of the pancreas

\begin{tabular}{lccccc}
\hline & LD/PanIN-1 and -2 & HD/PanIN-3 & IDC & LD/PanIN-1 and -2 vs HD/PanIN-3 & HD/PanIN-3 vs IDC $^{\mathrm{a}}$ \\
\hline DUSP6 & $0 / 38(0 \%)$ & $0 / 24(0 \%)$ & $30 / 57(53 \%)$ & NA $^{\mathrm{b}}$ & $<0.0001$ \\
CDKN2A & $12 / 38(32 \%)$ & $20 / 24(83 \%)$ & $47 / 57(83 \%)$ & $<0.0001$ & $>0.9999$ \\
TP53 & $0 / 38(0 \%)$ & $10 / 24(42 \%)$ & $39 / 57(68 \%)$ & $<0.0001$ & 0.0451 \\
SMAD4 & $1 / 38(3 \%)$ & $13 / 24(54 \%)$ & $52 / 57(91 \%)$ & $<0.0001$ & 0.0003 \\
\hline
\end{tabular}

${ }^{\text {a } F i s h e r ' s ~ e x a c t ~} P$-value by $\chi^{2}$-test.

${ }^{\mathrm{b}} \mathrm{NA}$, not applicable.

LD, low-grade dysplasia; HD, high-grade dysplasia; PanIN, pancreatic intraepithelial neoplasia; IDC, invasive ductal carcinoma.

between low-grade dysplasia/pancreatic intraepithelial neoplasia-1 and -2 and high-grade dysplasia/pancreatic intraepithelial neoplasia-3 for CDKN2A, TP53, and SMAD4 $(P<0.0001$ for all $)$. DUSP6 is not applicable for this comparison because none of them revealed abnormality in both of the lesions. In comparison between high-grade dysplasia/pancreatic intraepithelial neoplasia-3 and the invasive carcinoma, significant differences were observed in DUSP6 $(P<0.0001)$ as well as in SMAD4 $(P=0.0003)$. None of the molecules statistically correlated with each other in any grade. These results indicated that gradual accumulation of inactivations of the molecules were associated with progression of the pathway through pancreatic intraepithelial neoplasia to invasive ductal carcinoma along the atypical grades; CDKN2A, TP53, and SMAD4 were associated with the progression from low-grade dysplasia/pancreatic intraepithelial neoplasia-1 and -2 to high-grade dysplasia/pancreatic intraepithelial neoplasia-3, while the inactivation of DUSP6 was strongly associated with the progression from high-grade dysplaisa/pancreatic intraepithelial neoplasia-3 to invasive carcinoma.

In the retrieved lesions from pancreata with intraductal papillary-mucinous neoplasms, abnormal expressions of DUSP6 as well as CDKN2A, TP53, SMAD4 were analyzed; results are summarized in Table 2 and Figure 1b. In comparisons among atypical grades, significant differences were observed between intraductal papillary-mucinous adenoma/borderlines and intraductal papillary-mucinous carcinomas for CDKN2A $(P=0.0188)$ and TP53 $(P=0.0159)$ and between intraductal papillary-mucinous carcinomas and the invasive components of invasive ductal carcinoma associated with intraductal papillary-mucinous neoplasm for SMAD4 $(P=0.0148)$. None of the molecules statistically correlated with each other in any grade.

Morphological variations of papillae in the intraductal papillary-mucinous neoplasms were evaluated and classified according to criteria based on morphologies and expressions of mucin proteins including MUC1-CORE, MUC2, and MUC5AC, as described elsewhere, ${ }^{34}$ as follows: 21 cases of the gastric (null) type, 26 cases of the intestinal type, three cases of the pancreatobiliary type, and one case of the oncocytic type. The evaluated types and abrogations of DUSP6, CDKN2A, TP53, and SMAD4 were statistically compared, but no significant associations were observed.

Since the underexpression of DUSP6 was observed in a relatively small fraction of intraductal papillary-mucinous adenoma/borderlines (8/47, $17 \%$ ), we investigated mutations of KRAS2 in those lesions to determine whether or not the underexpression would be pathologic. First, we investigated the mutation of codon 12 of KRAS2 by the allele-specific oligonucleotide hybridization analysis in six of the eight of intraductal papillarymucinous adenoma/borderlines with underexpressions of DUSP6. We found that five of these 6 lesions harbored KRAS2 mutations. The samples revealing no mutation by the allele-specific oligonucleotide hybridization were submitted for additional analyses of codons 12, 13, and 61 by the direct nucleotide sequencing, which resulted in no additional finding of mutations. These results indicated that most of the lesions of intraductal papillarymucinous adenoma/borderlines with underexpressions of DUSP6 were pathologic in phenotypes (Figure 3, Table 3).

In order to understand the differences between the molecular pathways involved in pancreatic intraepithelial neoplasia and intraductal papillary-mucinous neoplasms, the frequencies of abnormal expressions were compared. As shown in Table 4, the abnormal expression of DUSP6 was significantly less frequently observed in low-grade dysplasia/ pancreatic intraepithelial neoplasia-1 and -2 than in intraductal papillary-mucinous adenoma/borderlines $(P=0.0076)$. The abnormal expression of SMAD4 was significantly more frequent in highgrade dysplasia/pancreatic intraepithelial neoplasia-3 than in intraductal papillary-mucinous carcinomas $(P<0.0001)$. The abnormal expressions of SMAD4 and TP53 were significantly more frequent in invasive ductal carcinomas than in the invasive components of colloid mucinous carcinoma associated with intraductal papillary-mucinous neoplasms $(P=0.0011$ and 0.0493 , respectively). The abnormal expressions of TP53 were significantly more frequent in invasive ductal carcinoma than in the invasive ductal components in invasive 
LD/PanlN-1, -2

IDC

HD/PanIN-3
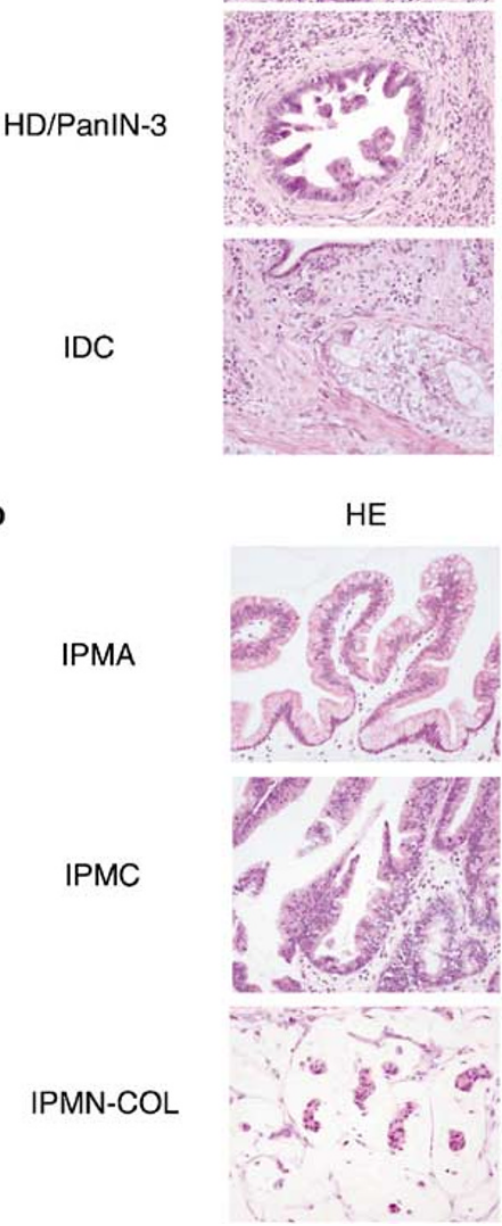

HE
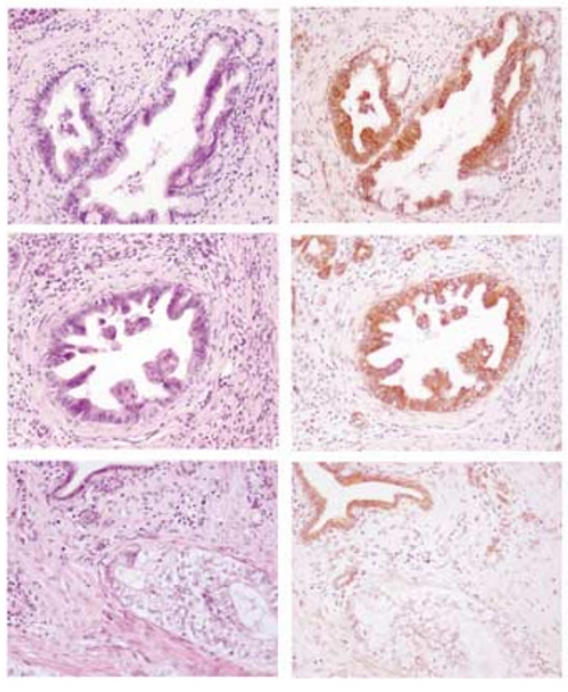

DUSP6
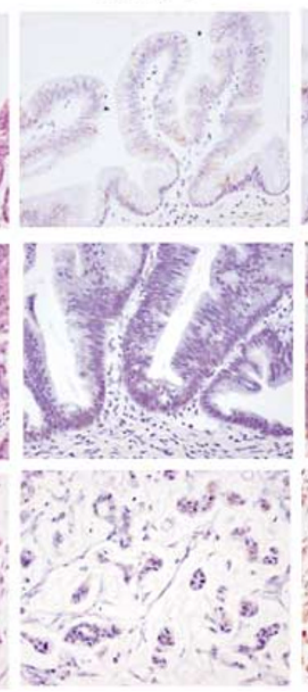

CDKN2A

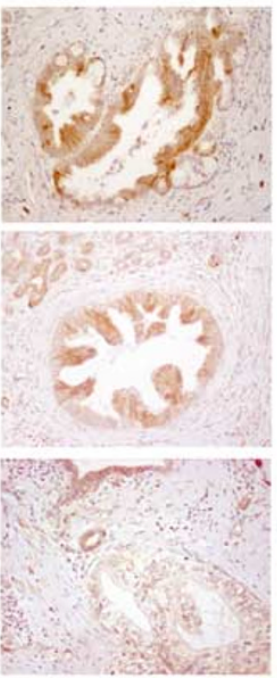

CDKN2A

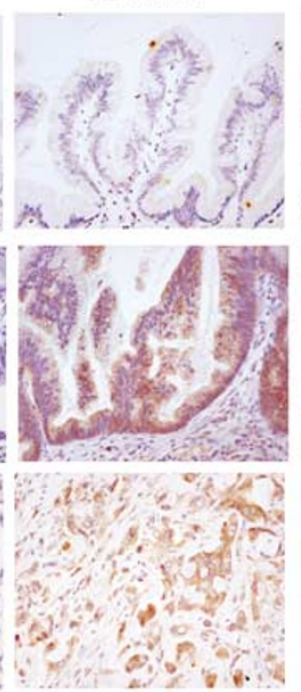

TP53
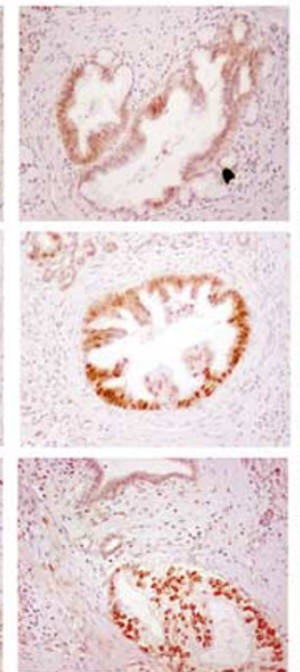

TP53



SMAD4
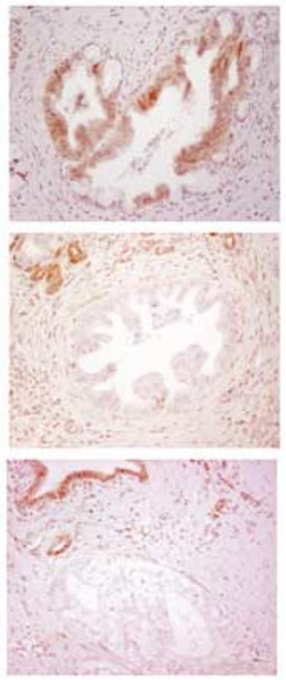

SMAD4

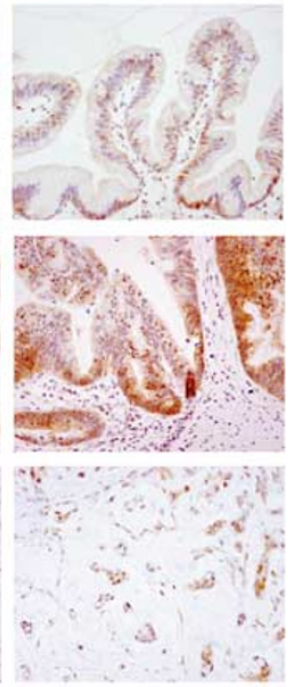

Figure 1 Representative images of immunohistochemistry for DUSP6, CDKN2A, TP53, and SMAD4 in low-grade dysplasia/pancreatic intraepithelial neoplasia-1 and -2 (LD/PanIN-1, -2), high-grade dysplasia/pancreatic intraepithelial neoplasia-3 (HD/PanIN-3), and invasive ductal carcinoma (IDC) (a) and in intraductal papillary-mucinous adenoma (IPMA), intraductal papillary-mucinous carcinoma (IPMC), and invasive colloid mucinous carcinoma associated with intraductal papillary-mucinous neoplasm (IPMN-COL) (b). Panel a shows overexpression of DUSP6 in both low-grade dysplasia/pancreatic intraepithelial neoplasia-1 and -2 and high-grade dysplasia/ pancreatic intraepithelial neoplasia-3 in contrast with its underexpression in invasive ductal carcinoma along with loss of intranuclear expression of CDKN2A throughout the lesions, abnormal intranuclear accumulation of TP53 and loss of expression of SMAD4 in highgrade dysplasia/pancreatic intraepithelial neoplasia-3 and invasive ductal carcinoma. Panel b shows underexpression of DUSP6 in intraductal papillary-mucinous adenoma, intraductal papillary-mucinous carcinoma, and invasive colloid mucinous carcinoma associated with intraductal papillary-mucinous neoplasm along with loss of intranuclear expression of CDKN2A in all the lesions, abnormal intranuclear accumulation of TP53 in intraductal papillary-mucinous carcinoma, and constitutive expressions of SMAD4 throughout the lesions.

ductal carcinoma associated with intraductal papillary-mucinous neoplasms $(P=0.0389)$, although the number of examined cases for the latter was quite small (three cases).

\section{Discussion}

In this study, we found that abrogation of DUSP6 was significantly associated with progression from pancreatic intraepithelial neoplasia to the invasive ductal carcinoma, which was quite characteristic and independent of abrogations of other major tumor suppressor genes; the abrogation of CDKN2A was associated with the development of low-grade dysplasia/pancreatic intraepithelial neoplasia-1 and -2 , and abrogations of TP53 and SMAD4 were associated with the development of high-grade dysplasia/pancreatic intraepithelial neoplasia-3. The abrogation of SMAD4 was also significantly 
associated with the invasive phenotype, which was consistent with our previous results demonstrating the suppression of invasiveness after introduction of $S M A D 4$ in SMAD4-null pancreatic cancer cells. ${ }^{35}$

a

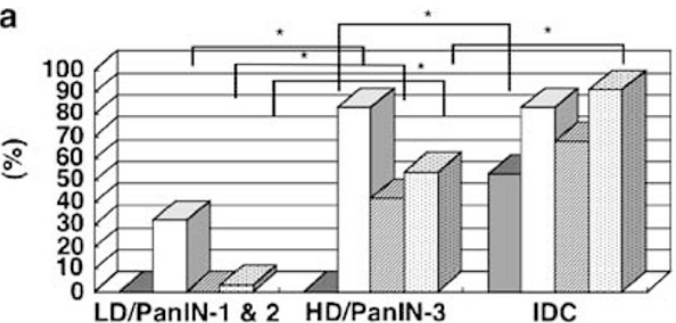

- DUSP6

$\square$ CDKN2A

$\square$ TP53

$\square$ SMAD4

" $p<0.001$

b

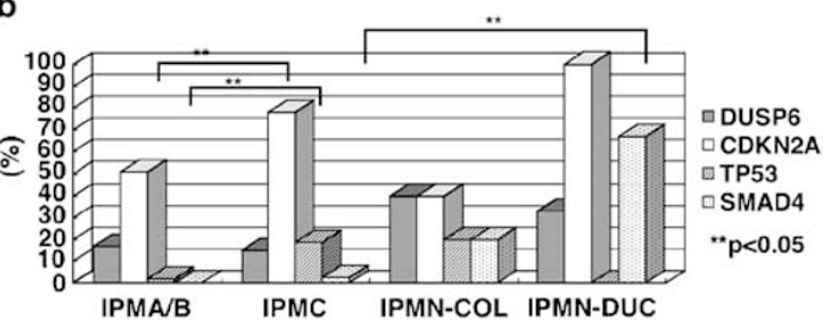

Figure 2 Frequency of inactivation of DUSP6, CDKN2A, TP53, and SMAD4 in pancreata with ductal dysplasia/pancreatic intraepithelial neoplasia (PanIN) and invasive ductal carcinoma (IDC) (a) and those with intraductal papillary-mucinous adenoma/borderline (IPMA/B), intraductal papillary-mucinous carcinoma (IPMC), invasive colloid mucinous carcinoma associated with intraductal papillary-mucinous neoplasm (IPMN-COL), and invasive ductal carcinoma associated with intraductal papillarymucinous neoplasm (IPMN-DUC) (b). Asterisks denote significant differences between the groups statistically evaluated by $\chi^{2}$ test.
However, no significant association was observed between the abrogation of DUSP6 and the abrogation of SMAD4 in spite of our previous results showing the frequent concordant losses of $12 q$ and $18 q$ in

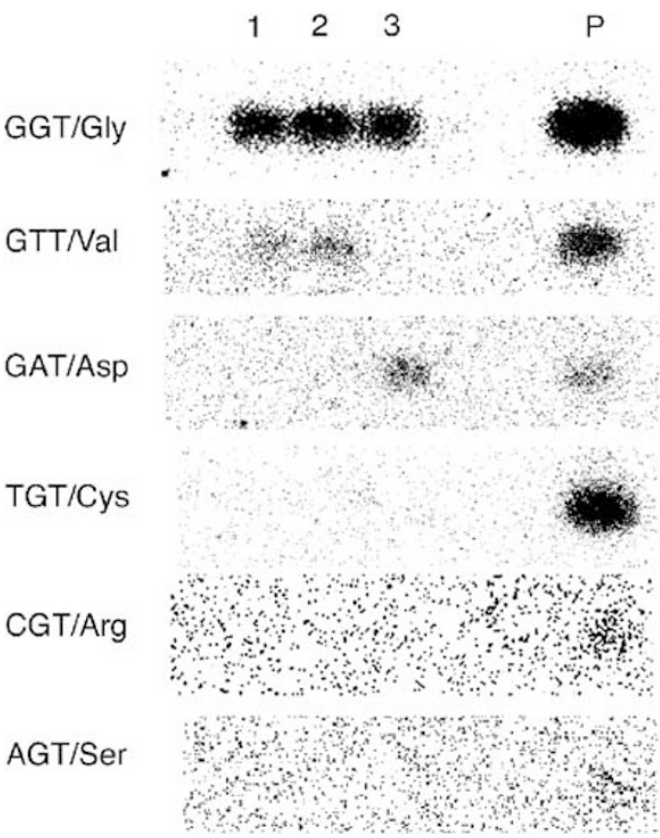

Figure 3 Representative examples of allele-specific oligonucleotide hybridization for analysis of KRAS2 mutation. Lanes 1-3 correspond to cases 4597,4868 , and 6973 , respectively. P denotes positive controls for each hybridization; PCR products prepared from genomic DNA of a normal human individual for GGT/Gly, PAN03JCK for GTT/Val, SU 86.86 for GAT/Asp, MIA PaCa-2 for TGT/Cys, PK-8 for CGT/Arg, and A549 for AGT/Ser.

Table 2 Abnormal expressions of DUSP6, CDKN2A, TP53, and SMAD4 in components of intraductal papillary-mucinous neoplasms

\begin{tabular}{|c|c|c|c|c|c|c|c|}
\hline & $I P M A / B$ & $I P M C$ & $I P M N-C O L$ & $I P M N-D U C$ & $I P M A / B$ vs $I P M C^{a}$ & $I P M C$ vs $I P M N-C O L^{\mathrm{a}}$ & $I P M C$ vs $I P M N-D U C^{a}$ \\
\hline DUSP6 & $8 / 47(17 \%)$ & $5 / 32(15 \%)$ & $2 / 5(40 \%)$ & $1 / 3(33 \%)$ & $>0.9999$ & 0.2330 & 0.4417 \\
\hline CDKN2A & $24 / 47(51 \%)$ & $25 / 32(78 \%)$ & $2 / 5(40 \%)$ & $3 / 3(100 \%)$ & 0.0188 & 0.1102 & $>0.9999$ \\
\hline TP53 & $1 / 47(2 \%)$ & 6/32 (19\%) & $1 / 5(20 \%)$ & $0 / 3(0 \%)$ & 0.0159 & $>0.9999$ & $>0.9999$ \\
\hline SMAD4 & $0 / 47(0 \%)$ & $1 / 32(3 \%)$ & $1 / 5(20 \%)$ & $2 / 3(67 \%)$ & 0.1571 & 0.2553 & 0.0148 \\
\hline
\end{tabular}

${ }^{\text {a } F i s h e r ' s ~ e x a c t ~} P$-value by $\chi^{2}$-test.

IPMA/B, intraductal papillary-mucinous adenoma/borderline; IPMC, intraductal papillary-mucinous carcinoma; IPMN-COL, invasive colloid mucinous carcinoma associated with intraductal papillary-mucinous neoplasms; IPMN-DUC, invasive ductal carcinoma associated with intraductal papillary-mucinous neoplasms.

Table 3 KRAS2 Mutation in intraductal papillary-mucinous adenoma/borderlines with abrogation of DUSP6

IPMA/B Sample\#

KRAS2

DUSP6 expression

\begin{tabular}{lll}
\hline Codon 12 & Codon 13 & Codon 61
\end{tabular}

\begin{tabular}{lllr}
\hline 179166 & GAT/Asp & & Faint/reduced \\
291 & GGT/Gly (wild type) & GGC/Gly (wild type) & Faint/reduced \\
4597 & GTT/Val & & Faint/reduced \\
6973 & GAT/Asp & Faint/reduced \\
4868 & GTT/Val & Faint/reduced \\
132115 & GTT/Val & Faint/reduced
\end{tabular}

IPMA/B, intraductal papillary-mucinous adenoma/borderlines. 
Table 4 Comparison of abnormal expressions of DUSP6, CDKN2A, TP53, and SMAD4 between pancreatic intraepithelial neoplasiainvasive ductal carcinoma and intraductal papillary-mucinous neoplasms

\begin{tabular}{|c|c|c|c|c|}
\hline & $L D /$ PanIN-1 and -2 vs $I P M A / B$ & HD/PanIN-3 vs IPMC & $I D C$ vs $I P M N-C O L$ & $I D C$ vs $I P M N-D U C$ \\
\hline DUSP6 & $\begin{array}{c}0 / 38 \text { vs } 8 / 47 \\
(0.0076)\end{array}$ & $\begin{array}{c}0 / 24 \text { vs } 5 / 32 \\
(0.0638)\end{array}$ & $\begin{array}{c}30 / 57 \text { vs } 2 / 5 \\
(0.6666)\end{array}$ & $\begin{array}{c}30 / 57 \text { vs } 1 / 3 \\
(0.6059)\end{array}$ \\
\hline CDKN2A & $\begin{array}{c}12 / 38 \text { vs } 27 / 47 \\
(0.0815)\end{array}$ & $\begin{array}{c}20 / 24 \text { vs } 25 / 32 \\
(0.7413)\end{array}$ & $\begin{array}{c}47 / 57 \text { vs } 2 / 5 \\
(0.0576)\end{array}$ & $\begin{array}{c}47 / 57 \text { vs } 3 / 3 \\
(>0.9999)\end{array}$ \\
\hline TP53 & $\begin{array}{c}0 / 38 \text { vs } 1 / 47 \\
(>0.9999)\end{array}$ & $\begin{array}{c}10 / 24 \text { vs } 6 / 32 \\
(0.0777)\end{array}$ & $\begin{array}{c}39 / 57 \text { vs } 1 / 5 \\
(0.0493)\end{array}$ & $\begin{array}{c}39 / 57 \text { vs } 0 / 3 \\
(0.0389)\end{array}$ \\
\hline SMAD4 & $\begin{array}{c}1 / 38 \text { vs } 0 / 47 \\
(0.4270)\end{array}$ & $\begin{array}{c}13 / 24 \text { vs } 1 / 32 \\
(<0.0001)\end{array}$ & $\begin{array}{c}52 / 57 \text { vs } 1 / 5 \\
(0.0011)\end{array}$ & $\begin{array}{c}52 / 57 \text { vs } 2 / 3 \\
(0.2752)\end{array}$ \\
\hline
\end{tabular}

Numbers in parentheses denote Fisher's exact $P$-value by $\chi^{2}$-test.

LD, low-grade dysplasia; HD, high-grade dysplasia; PanIN, pancreatic intraepithelial neoplasia; IDC, invasive ductal carcinoma; IPMA/B, intraductal papillary-mucinous adenoma/borderline; IPMC, intraductal papillary-mucinous carcinoma; IPMN-COL, invasive colloid mucinous carcinoma associated with intraductal papillary-mucinous neoplasms; IPMN-DUC, invasive ductal carcinoma associated with intraductal papillary-mucinous neoplasms.

invasive pancreatic cancer. ${ }^{36}$ These results suggest that both molecules may associate with the invasive phenotype independently, that is, RAS-MAPK pathway vs TGFB-SMAD pathway, and another molecules might be involved concordantly in invasive phenotypes. The significant association between the abrogation of DUSP6 and the invasive phenotype suggests that DUSP6 serves as a gatekeeper for the progression from pancreatic intraepithelial neoplasia to invasive ductal carcinomas. Fairly preserved/ overexpressions of DUSP6 in pancreatic intraepithelial neoplasia are suggested to be a result of accelerating feedback mechanism of suppression of hyperactivated/mutated RAS. Breakdown of the feedback loop indicated by the abrogated expression of DUSP6 may have contributed to progression from pancreatic intraepithelial neoplasia to invasive ductal carcinoma. As we reported previously in the experiment employing cultured pancreatic cancer cells, the abrogation of DUSP6 seems to be associated with the constitutive activation/phosphorylation of MAPK1 synergistically under a gain-of-function mutation of KRAS $2,{ }^{8}$ which may play a major role in the establishment of invasive ductal carcinoma of the pancreas. Our results indicated that inactivations of CDKN2A, TP53, and SMAD4 were associated with progression of pancreatic intraepithelial neoplasia from low-grade dysplasia to high-grade dysplasia, which was consistent with reports by others. ${ }^{18,37,38}$

We found that expressions of DUSP6 were abrogated in a relatively small fraction of intraductal papillary-mucinous adenoma/borderline lesions, most of which harbored the mutated KRAS2. As we described in the Introduction, we hypothesized that the inactivation of DUSP6 under mutated KRAS2 would induce a pathologic phenotype for the pancreatic carcinogenesis. We can assume that the hypothesis can be applied to initiation of some of intraductal papillary-mucinous neoplasms, although its frequency may be relatively low. However, the potential association of DUSP6 abrogation with an initiation of the intraductal papillarymucinous neoplasm is of quite different character from that in the pathway through pancreatic intraepithelial neoplasia to the invasive ductal carcinoma, in which DUSP6 abrogation was associated exclusively with the invasive phenotype. Our results suggest that DUSP6 may play a different role in the progression of intraductal papillary-mucinous neoplasms from that of pancreatic intraepithelial neoplasia to the invasive ductal carcinoma.

We also found that the abrogation of SMAD4 was significantly associated with the ductal invasive phenotype but not with the colloid mucinous invasive phenotype of intraductal papillary-mucinous neoplasms. Although the number of analyzed cases with invasive carcinomas associated with intraductal papillary-mucinous neoplasms was not large, our present results suggest that the invasive ductal phenotype and the invasive colloid mucinous phenotype may be results of progressions through distinct molecular pathways involving SMAD4.

Our recent results indicated that epigenetic mechanisms suppressed expression of DUSP6. ${ }^{39}$ However, upstream mechanism(s) for this suppression still remains in an open question.

\section{Acknowledgements}

Grant support: Grant-in-Aid from Ministry of Education, Culture, Sports, Science, and Technology of Japan, the Japan Health Sciences Foundation, Pancreas Research Foundation of Japan, Gonryo Medical Foundation, Vehicle Racing Commemorative Foundation, and Foundation for Promotion of Cancer Research in Japan. 
We thank Dr Barbara Lee Smith Pierce (Adjunct Professor, University of Maryland University College) for editorial work in the preparation of this manuscript.

\section{References}

1 Hahn SA, Seymour AB, Hoque AT, et al. Allelotype of pancreatic adenocarcinoma using xenograft enrichment. Cancer Res 1995;55:4670-4675.

2 Kimura M, Abe T, Sunamura M, et al. Detailed deletion mapping on chromosome arm $12 \mathrm{q}$ in human pancreatic adenocarcinoma: identification of a 1-cM region of common allelic loss. Genes Chromosomes Cancer 1996;17:88-93.

3 Fukushige S, Waldman FM, Kimura M, et al. Frequent gain of copy number on the long arm of chromosome 20 in human pancreatic adenocarcinoma. Genes Chromosomes Cancer 1997;19:161-169.

4 Caldas C, Hahn SA, da Costa LT, et al. Frequent somatic mutations and homozygous deletions of the p16 (MTS1) gene in pancreatic adenocarcinoma. Nat Genet 1994;8:27-32.

5 Redston MS, Caldas C, Seymour AB, et al. p53 mutations in pancreatic carcinoma and evidence of common involvement of homocopolymer tracts in DNA microdeletions. Cancer Res 1994;54:3025-3033.

6 Hahn SA, Schutte M, Hoque AT, et al. DPC4, a candidate tumor suppressor gene at human chromosome 18q21.1. Science 1996;271:350-353.

7 Furukawa T, Yatsuoka T, Youssef EM, et al. Genomic analysis of DUSP6, a dual specificity MAP kinase phosphatase, in pancreatic cancer. Cytogenet Cell Genet 1998;82:156-159.

8 Furukawa T, Sunamura M, Motoi F, et al. Potential tumor suppressive pathway involving DUSP6/MKP-3 in pancreatic cancer. Am J Pathol 2003;162:1807-1815.

9 Furukawa T, Horii A. Molecular pathology of pancreatic cancer: in quest of tumor suppressor genes. Pancreas 2004;28:253-256.

10 Muda M, Boschert U, Dickinson R, et al. MKP-3, a novel cytosolic protein-tyrosine phosphatase that exemplifies a new class of mitogen-activated protein kinase phosphatase. J Biol Chem 1996;271:4319-4326.

11 Groom LA, Sneddon AA, Alessi DR, et al. Differential regulation of the MAP, SAP and RK/p38 kinases by Pyst1, a novel cytosolic dual-specificity phosphatase. EMBO J 1996;15:3621-3632.

12 Tsang M, Maegawa S, Kiang A, et al. A role for MKP3 in axial patterning of the zebrafish embryo. Development 2004;131:2769-2779.

13 Kawakami Y, Rodriguez-Leon J, Koth CM, et al. MKP3 mediates the cellular response to FGF8 signalling in the vertebrate limb. Nat Cell Biol 2003;5:513-519.

14 Sommers SC, Murphy SA, Warren S. Pancreatic duct hyperplsia and cancer. Gastroenterology 1954;27: 629-640.

15 Cubilla AL, Fitzgerald PJ. Morphological lesions associated with human primary invasive nonendocrine pancreas cancer. Cancer Res 1976;36:2690-2698.

16 Kozuka S, Sassa R, Taki T, et al. Relation of pancreatic duct hyperplasia to carcinoma. Cancer 1979;43: 1418-1428.

17 Wilentz RE, Geradts J, Maynard R, et al. Inactivation of the p16 (INK4A) tumor-suppressor gene in pancreatic duct lesions: loss of intranuclear expression. Cancer Res 1998;58:4740-4744.

18 Wilentz RE, Iacobuzio-Donahue CA, Argani P, et al. Loss of expression of Dpc4 in pancreatic intraepithelial neoplasia: evidence that DPC4 inactivation occurs late in neoplastic progression. Cancer Res 2000; 60:2002-2006.

19 Furukawa T, Chiba R, Kobari M, et al. Varying grades of epithelial atypia in the pancreatic ducts of humans. Classification based on morphometry and multivariate analysis and correlated with positive reactions of carcinoembryonic antigen. Arch Pathol Lab Med 1994;118:227-234.

20 Hruban RH, Goggins M, Parsons J, et al. Progression model for pancreatic cancer. Clin Cancer Res 2000;6: 2969-2972.

21 Hruban RH, Adsay NV, Albores-Saavedra J, et al. Pancreatic intraepithelial neoplasia: a new nomenclature and classification system for pancreatic duct lesions. Am J Surg Pathol 2001;25:579-586.

22 Ohhashi K, Murakami Y, Takekoshi T. Four cases of 'mucin producing' cancer of the pancreas on specific findings of the papilla of Vater (Abstr). Prog Diagn Endosc 1982;20:348-351.

23 Furukawa T, Takahashi T, Kobari $\mathrm{M}$, et al. The mucus-hypersecreting tumor of the pancreas. Development and extension visualized by threedimensional computerized mapping. Cancer 1992;70: 1505-1513.

24 Adsay NV, Merati K, Andea A, et al. The dichotomy in the preinvasive neoplasia to invasive carcinoma sequence in the pancreas: differential expression of MUC1 and MUC2 supports the existence of two separate pathways of carcinogenesis. Mod Pathol 2002;15:1087-1095.

25 Iacobuzio-Donahue CA, Klimstra DS, Adsay NV, et al. Dpc-4 protein is expressed in virtually all human intraductal papillary mucinous neoplasms of the pancreas: comparison with conventional ductal adenocarcinomas. Am J Pathol 2000;157:755-761.

26 Adsay NV, Conlon KC, Zee SY, et al. Intraductal papillary-mucinous neoplasms of the pancreas: an analysis of in situ and invasive carcinomas in 28 patients. Cancer 2002;94:62-77.

27 Hruban RH, Takaori K, Klimstra DS, et al. An illustrated consensus on the classification of pancreatic intraepithelial neoplasia and intraductal papillary mucinous neoplasms. Am J Surg Pathol 2004;28: 977-987.

28 Kondo E, Furukawa T, Yoshinaga K, et al. Not hMSH2 but hMLH1 is frequently silenced by hypermethylation in endometrial cancer but rarely silenced in pancreatic cancer with microsatellite instability. Int J Oncol 2000;17:535-541.

29 Verlaan-de Vries M, Bogaard ME, van den Elst H, et al. A dot-blot screening procedure for mutated ras oncogenes using synthetic oligodeoxynucleotides. Gene 1986;50:313-320.

30 Sun C, Yamato T, Furukawa T, et al. Characterization of the mutations of the K-ras, p53, p16, and SMAD4 genes in 15 human pancreatic cancer cell lines. Oncol Rep 2001;8:89-92.

31 Mori Y, Shiwaku H, Fukushige S, et al. Alternative splicing of $h M S H 2$ in normal human tissues. Hum Genet 1997;99:590-595.

32 Mitchell CE, Belinsky SA, Lechner JF. Detection and quantitation of mutant K-ras codon 12 restriction 
fragments by capillary electrophoresis. Anal Biochem 1995;224:148-153.

33 Sakurada A, Suzuki A, Sato M, et al. Infrequent genetic alterations of the PTEN/MMAC1 gene in Japanese patients with primary cancers of the breast, lung, pancreas, kidney, and ovary. Jpn J Cancer Res 1997;88:1025-1028.

34 Adsay NV, Merati K, Basturk O, et al. Pathologically and biologically distinct types of epithelium in intraductal papillary mucinous neoplasms: delineation of an "intestinal" pathway of carcinogenesis in the pancreas. Am J Surg Pathol 2004;28:839-848.

35 Duda DG, Sunamura M, Lefter LP, et al. Restoration of SMAD4 by gene therapy reverses the invasive phenotype in pancreatic adenocarcinoma cells. Oncogene 2003;22:6857-6864
36 Yatsuoka T, Sunamura M, Furukawa T, et al. Association of poor prognosis with loss of $12 q, 17 p$, and 18q, and concordant loss of $6 q / 17 p$ and $12 q / 18 q$ in human pancreatic ductal adenocarcinoma. Am J Gastroenterol 2000;95:2080-2085.

37 Rozenblum E, Schutte M, Goggins M, et al. Tumorsuppressive pathways in pancreatic carcinoma. Cancer Res 1997;57:1731-1734.

38 Maitra A, Adsay NV, Argani P, et al. Multicomponent analysis of the pancreatic adenocarcinoma progression model using a pancreatic intraepithelial neoplasia tissue microarray. Mod Pathol 2003;16: 902-912.

39 Xu S, Furukawa T, Kanai N, et al. Abrogation of DUSP6 by hypermethylation in human pancreatic cancer. J Hum Genet, in press. 